\title{
Endodontia minimamente invasiva: uma revisão de literatura
}

\author{
Minimally invasive endodontics: a literature review \\ Endodoncia mínimamente invasiva: una revisión de la literatura
}

Recebido: 27/10/2021 | Revisado: 06/11/2021 | Aceito: 10/11/2021 | Publicado: 15/11/2021

\author{
Laisla Siqueira Barros Lopes \\ ORCID: https://orcid.org/0000-0002-1959-9047 \\ Faculdade Independente do Nordeste, Brasil \\ E-mail: laislalopes30@gmail.com \\ Franciele Meira Coelho \\ ORCID: https://orcid.org/0000-0003-2380-0791 \\ Faculdade Independente do Nordeste, Brasil \\ E-mail: franmc112233@gmail.com \\ Polyana Argolo Souza Amaral \\ ORCID: https://orcid.org/0000-0002-4742-5562 \\ Faculdade Independente do Nordeste, Brasil \\ E-mail: polyana.argolo@gmail.com \\ Lara Correia Pereira \\ ORCID: https://orcid.org/0000-0002-5529-9260 \\ Faculdade Independente do Nordeste, Brasil \\ E-mail: laucpereira@hotmail.com
}

\begin{abstract}
Resumo
A endodontia é a especialidade da odontologia que se dedica as alterações da polpa dental e dos tecidos periapicais e busca analisar sua fisiologia, morfologia e patologia, incluindo a biologia da polpa normal, a etiologia, o diagnóstico, a prevenção e o tratamento das patologias e injúrias que atingem a polpa, relacionadas ou não, às alterações no periápice. O tratamento endodôntico tradicional vem sendo argumentado pelos profissionais após o surgimento do termo tratamento endodôntico minimamente invasivo, no qual prioriza não apenas remover ou reverter à patologia, mas também preservar a estrutura dental sadia, promovendo uma maior resistência da estrutura dentária. O presente trabalho tem como objetivo revisar a literatura investigando o acesso endodôntico minimamente invasivo e suas possíveis repercussões, impactos e consequências no tratamento endodôntico. Foi realizado um levantamento bibliográfico nas bases de dados Google Acadêmico, PubMed, Scielo e Bireme, onde foram selecionados artigos publicados em inglês e português. Diante do exposto, a endodontia minimamente invasiva não traz segurança para o tratamento endodôntico, tampouco resistência à fratura do elemento dental, estudos comprovaram a deficiência nas demais etapas subsequentes do tratamento após abordagens minimamente invasivas, como na desinfecção e modelagem dos canais radiculares e posterior obturação. $\mathrm{O}$ acesso contemporâneo é uma alternativa que vem sendo estudada para trazer uma melhora ao acesso convencional sem causar danos nas demais etapas, pois é conduzido pela anatomia e morfologia do sistema de canais radiculares que objetiva a preservação de estrutura dentária sem desconsiderar bases científicas e biológicas da endodontia.
\end{abstract}

Palavras-chave: Endodontia; Cavidade pulpar; Tratamento conservador.

\begin{abstract}
Endodontics is the specialty of dentistry that is dedicated to changes in dental pulp and periapical tissues and seeks to analyze their physiology, morphology and pathology, including the biology of normal pulp, etiology, diagnosis, prevention and treatment of pathologies and injuries that reach the pulp, related or not to alterations in the periapex. The traditional endodontic treatment has been argued by professionals after the emergence of the term minimally invasive endodontic treatment, which prioritizes not only removing or reversing the pathology, but also preserving the healthy tooth structure, promoting greater resistance of the tooth structure. The minimally invasive endodontic approach proposes the geometric shape to be similar to the conventional one, the wear on the coronary portion is restricted by the pericervical dentin. One of the requirements to perform the minimally invasive access is the knowledge of the anatomy and morphology of the tooth to be treated, before performing the access, it is necessary to remove cavities and insufficient restorations. The objective of this work is to elaborate a literature review of a narrative nature, investigating the minimally invasive endodontic access and its repercussion, impact, consequences in the endodontic treatment. This is a literature review, based on the collection of scientific articles, carried out in worldwide bibliographic databases: Academic Google, PubMed, Scielo and Bireme. Articles published in English and Portuguese were selected. Minimally invasive endodontics is not safe for endodontic treatment, nor is it resistant to fracture of the dental element, studies have shown the deficiency in other subsequent stages of treatment after minimally invasive approaches, such as disinfection and shaping of root canals and subsequent filling. Contemporary
\end{abstract}


access is an alternative that has been studied to improve the conventional access without causing damage in the other stages, as it is guided by the anatomy and morphology of the root canal system that aims to preserve the tooth structure without disregarding the scientific and biological bases of the endodontics.

Keywords: Endodontics; Dental pulp cavity; Conservative treatment.

\section{Resumen}

La endodoncia es la especialidad de la odontología que se dedica a los cambios en la pulpa dental y los tejidos periapicales y busca analizar su fisiología, morfología y patología, incluyendo la biología de la pulpa normal, etiología, diagnóstico, prevención y tratamiento de patologías y lesiones que llegan a la pulpa. , relacionado o no con alteraciones en el periapex. El tratamiento de endodoncia tradicional ha sido argumentado por profesionales tras la aparición del término tratamiento de endodoncia mínimamente invasivo, que prioriza no solo eliminar o revertir la patología, sino también preservar la estructura dental sana, promoviendo una mayor resistencia de la estructura dentaria. El abordaje endodóntico mínimamente invasivo propone que la forma geométrica sea similar a la convencional, el desgaste en la porción coronaria está restringido por la dentina pericervical. Uno de los requisitos para realizar el acceso mínimamente invasivo es el conocimiento de la anatomía y morfología del diente a tratar, antes de realizar el acceso es necesario remover caries y restauraciones insuficientes. El objetivo de este trabajo es elaborar una revisión de la literatura de carácter narrativo, investigando el acceso endodóntico mínimamente invasivo y su repercusión, impacto, consecuencias en el tratamiento endodóntico. Se trata de una revisión de la literatura, basada en la colección de artículos científicos, realizada en 4 bases de datos bibliográficas mundiales: Google Académico, PubMed, Scielo y Bireme. Se seleccionaron artículos publicados en inglés y portugués. La endodoncia mínimamente invasiva no es segura para el tratamiento de endodoncia, ni es resistente a la fractura del elemento dental, estudios han demostrado la deficiencia en otras etapas posteriores del tratamiento luego de abordajes mínimamente invasivos, como la desinfección y conformación de conductos radiculares y posterior obturación. El acceso contemporáneo es una alternativa que se ha estudiado para mejorar el acceso convencional sin causar daño en las otras etapas, ya que se guía por la anatomía y morfología del sistema de conductos radiculares que tiene como objetivo preservar la estructura dentaria sin desconocer las bases científicas y biológicas. de la endodoncia.

Palabras clave: Endodoncia; Cavidad pulpar; Tratamiento conservador.

\section{Introdução}

O tratamento endodôntico tem como principal finalidade a conservação em longo prazo de um dente em função, prevenindo ou tratando patologias pulpares e/ou periapicais. O tratamento endodôntico tradicional vem sendo argumentado pelos profissionais após o surgimento do termo tratamento endodôntico minimamente invasivo, no qual prioriza não apenas remover ou reverter à patologia, mas também preservar a estrutura dental sadia o que promove maior resistência a fraturas e evita a precoce perda do elemento dentário (Carvalho, Monteiro, Santo, \& Porto, 2020).

A cirurgia de acesso convencional propõe uma boa localização dos canais radiculares, dessa forma facilitando então, o preparo químico-mecânico e posterior obturação (Silva, \& Silva, 2019). Mesmo que o acesso endodôntico minimamente invasivo (AEMI) propõe a forma geométrica similar a da convencional, o desgaste na porção coronária se restringe pela dentina pericervical (Carvalho, Monteiro, Santo, \& Porto, 2020). A dentina situada próxima à crista óssea alveolar é encarregada de transferir a carga oclusal para a raiz do elemento dental (Silva, \& Silva, 2019).

Acessos tradicionais que contornam toda a circunferência da câmara pulpar acabam resultando em um desgaste de $50 \%$ a mais que o necessário, resultando na perda de estrutura dentária sadia. Os erros no acesso são originados em aberturas além ou aquém do tamanho necessário, acessos pequenos podem prejudicar a localização dos canais e acessos grandes podem causar remoção desnecessária de estrutura dentária e causar enfraquecimento do remanescente coronário, podendo causar danos irreparáveis em longo prazo (Vasconcellos et al., 2012).

Apesar dos estudos microbiológicos não comprovarem o tamanho necessário do preparo para que haja uma ação antimicrobiana eficaz, existe um atrito entre abordagens minimamente invasivas e a desinfecção dos canais radiculares, uma vez que acessos muito pequenos podem acabar prejudicando a limpeza e desinfecção do sistema de canais radicular (Gluskin, Peters, \& Peters, 2014).

Um dos requisitos para realizar o AEMI é o conhecimento da anatomia e morfologia do dente a ser tratado, por isso, é de suma importância uma boa radiografia, pois a mesma fornecerá uma orientação inicial em relação à localização dos canais 
radiculares. Antes de realizar o acesso, é necessário que seja feito a remoção de cáries e restaurações insuficientes (Bürklein, \& Schäfer, 2015).

Embora não existam evidências científicas consolidadas na literatura que comprove os benefícios da técnica do AEMI em longo prazo, esse tipo de tratamento já vem sendo executado na endodontia (Carvalho, Monteiro, Santo, \& Porto, 2020). Dessa forma, o presente trabalho tem como objetivo revisar a literatura investigando o acesso endodôntico minimamente invasivo e suas possíveis repercussões, impactos e consequências no tratamento endodôntico.

\section{Metodologia}

Este estudo trata-se de uma revisão de literatura de cunho narrativo e bibliográfico de acordo com a metodologia de Silva (2019), baseado na coleta de dados sobre o tratamento endodôntico minimamente invasivo, enfatizando as cavidades de acesso endodônticos. Foram selecionados artigos compreendidos entre os anos de 2010 a 2021, escritos na língua portuguesa e inglesa publicados em revistas nacionais e internacionais.

A coleta e seleção dos artigos científicos para elaboração deste trabalho foi realizada através das principais bases de dados: Google Acadêmico, PubMed, Scielo e Bireme, com o auxílio dos seguintes descritores de saúde (DeCS): endodontia, cavidade pulpar, tratamento conservador. Os critérios de inclusão para escolha dos artigos foram: revisões de literatura e artigos que abordam o tema. Os critérios analisados para a exclusão dos artigos são: artigos publicados antes de 2010, artigos escritos em idiomas distintos do inglês e português.

Foram encontrados 31 artigos no total, os quais foram avaliados por meio do título, resumo e de seu texto na íntegra. Após remoção de artigos duplicados e aplicar os critérios de inclusão, restaram 20 artigos lidos e analisados. Foi realizado e coletado o material necessário, separando-os em pertinentes ou não ao tema, avaliando a visão de cada autor, baseados em evidências científicas.

\section{Revisão de Literatura}

A endodontia é a especialidade da odontologia que se dedica das alterações da polpa dental e dos tecidos periapicais e busca analisar sua fisiologia, morfologia e patologia, incluindo a biologia da polpa normal, a etiologia, o diagnóstico, a prevenção e o tratamento das patologias e injúrias que atingem a polpa, relacionadas ou não, às alterações no periápice. Portanto, o tratamento endodôntico tem como principal finalidade a conservação em longo prazo de um dente em função, prevenindo ou tratando patologias pulpares e/ou periapicais (Carvalho, Monteiro, Santo, \&amp; Porto, 2020).

Tratamentos minimamente invasivos estão sendo adotados em todos os âmbitos da área da saúde em decorrência dos avanços na engenharia de microssistemas, nanotecnologia, terapia a laser e ferramentas de imagem de alta resolução para diagnóstico e orientação de instrumentos cirúrgicos. Na odontologia não vem sendo diferente, por exemplo, há um enorme empenho em novos meios de tratar a doença cárie, preservando o máximo de estrutura dentária. Na Endodontia, uma proposta marcante foi feita por Clark e Khademi (2010), que defendeu a conservação da dentina durante o acesso endodôntico e instrumentação do canal radicular baseando nas seguintes justificativas: (I) o raciocínio lógico que afirma que a perda total de massa dentinária inevitavelmente reduziria a capacidade do dente de resistir às forças mastigatórias intermitentes em longo prazo e (II) nenhum material sintético pode substituir adequadamente o tecido dentinário perdido (Silva, Versiani, Souza, \& De-Deus, 2020).

A forma dos acessos endodônticos convencionais (AEC), para os diferentes grupos dentários, se manteve o mesmo por anos com apenas pequenas modificações, promovendo a remoção controlada da estrutura dentária. O AEC tem como objetivo remover todo o teto da câmara pulpar, possibilitando uma visão direta e um acesso reto e livre até a primeira curvatura do canal, facilitando a penetração do instrumento sem nenhuma interferência facilitando a desinfecção, modelagem e posterior 
obturação dos canais radiculares, podendo assim prevenir complicações durante o tratamento endodôntico (Boveda, \& Kishen, 2015; Mukherjee, Patel Chandak, \& Kashikar, 2017).

Segundo Buchanan, acessos tradicionais que contornam toda a circunferência da câmara pulpar acabam resultando em um desgaste de $50 \%$ a mais que o necessário, resultando na perda de estrutura dentária sadia (Vasconcellos et al., 2012). Os erros no acesso são originados em aberturas além ou aquém do tamanho necessário, acessos pequenos podem prejudicar a localização dos canais e acessos grandes, podem causar remoção desnecessária de estrutura dentária e causar enfraquecimento do remanescente coronário, todavia, a remoção da estrutura dentária hígida para a realização do preparo convencional pode diminuir e comprometer a resistência do elemento dentário quando submetidos a cargas funcionais (Tzimpoulas, Alisafis, \& Tzanetakis, 2012).

Num estudo prospectivo, os autores demonstram a importância de que ocorra um aprimoramento da técnica endodôntica existente e o surgimento de novas técnicas que promova uma maior longevidade do dente em função. À vista disso, atualmente, outros tipos de acessos endodônticos têm sido discutido na literatura: o acesso minimamente invasivo, o acesso ultraconservador, também citado como "ninja", o acesso dividido ou treliça e o acesso contemporâneo (Carvalho, Monteiro, Santo, \& Porto, 2020).

$\mathrm{O}$ acesso conservador visa à remoção mínima da estrutura dentária, preservando parcialmente o teto da câmara pulpar e a dentina pericervical, situada próxima a crista óssea alveolar (Silva et al., 2017), o acesso ultraconservador segue os mesmos princípios, porém a cavidade será ainda menor, enquanto as cavidades de acesso em treliça ou dividido segue uma abordagem distinta do conceito de AEMI, o acesso dividido envolve cavidades individuais separadas em dentes com mais raízes, portanto é feito um orifício em direção a cada corno pulpar, para maximizar a preservação da dentina entre as cavidades (Barbosa et al., 2020) e o acesso contemporâneo que é conduzido pela anatomia do sistema de canais radiculares promovendo maior preservação do tecido dentário sem desprezar fundamentos científicos e biológicos da endodontia, como a remoção total do teto da câmara pulpar assegurando uma melhor desinfecção no preparo químico-mecânico e promove a utilização dos instrumentos sem interferência coronárias (Silva et al., 2021).

Para realizar tais acessos é imprescindível o conhecimento da anatomia e morfologia do dente a ser tratado, por isso, é de suma importância uma boa radiografia, pois a mesma fornecerá uma orientação inicial em relação à localização dos canais radiculares. Antes de realizar o acesso, é necessário que seja feito a remoção de cáries e restaurações insuficientes (Bürklein, \& Schäfer, 2015).

O conceito de endodontia minimamente invasiva (EMI) vem ganhando gradativamente mais atenção. Seu principal objetivo é permitir a prevenção ou tratamento de doenças preservando tecidos e criando danos mínimos ao tratamento do canal radicular, mantendo assim a maior estrutura dentária possível. A questão dos AEMI, certamente, dominou as discussões recentes na literatura. No entanto, o conceito por trás da EMI é mais amplo do que apenas a questão das cavidades de acesso endodôntico. Existem outros fatores a serem considerados ao rotular um tratamento como minimamente invasivo na endodontia, como a utilização de instrumentos com menores diâmetro, ou instrumentos confeccionados com diferentes formas geométricas e características metalúrgicas, que objetivam permitir uma melhor adaptação à morfologia do canal durante a sua modelagem (Lima et al., 2020).

Apesar dos estudos microbiológicos não comprovarem o tamanho necessário do preparo para que haja uma ação antimicrobiana eficaz, existe um atrito entre abordagens minimamente invasivas e a desinfecção dos canais radiculares, uma vez que acessos muito pequenos podem acabar prejudicando a limpeza e desinfecção do sistema de canais radicular, (Gluskin, Peters, \& Peters, 2014) resultando em um maior acúmulo de debris no interior dos canais, a instrumentação se torna mais difícil, (Silva et al., 2019) aumentando a tensão sobre os instrumentos endodônticos, pois prejudica o acesso livre e direto, com isso intensifica o risco a fratura destes instrumentos, (Rover et al., 
2017) dificulta inclusive a obturação, principalmente em dentes multirradiculares e prejudica a estética caso a limpeza da câmara pulpar seja comprometida deixando resíduos de material obturador, dessa forma, resulta no aumento do tempo total do tratamento endodôntico. (Silva et al., 2019).

Embora não existam evidências científicas consideráveis na literatura que comprove os benefícios da técnica do AEMI em longo prazo esse tipo de tratamento já vem sendo executado na endodontia (Carvalho, Monteiro, Santo, \& Porto, 2020).

\section{Discussão}

A Endodontia deve ser uma especialidade com embasamento científico, ou seja, não pode levar em consideração puro raciocínio dedutivo. Devido os efeitos desfavoráveis das cavidades de AEMI relacionadas à localização do orifício do canal e a qualidade do preparo químico-mecânico do canal, os benefícios parecem ser ofuscados.

Para Carvalho, Monteiro, Santo, e Porto (2020), o AEMI quando comparado com os preparos endodônticos convencionais não promovem maior preservação da estrutura dentária e não promove aumento da resistência a fratura. Corroborando com Plotino et al., (2017) onde em um estudo recente mostrou que a cavidade endodôntica conservadora (CEC) não aumentou a resistência à fratura de molares maxilares restaurados em comparação com os dentes preparados com cavidade endodôntica tradicional (CET), sendo assim, não existiu benefício aparente da CEC relacionado à fratura.

Marinescu et al., (2020) realizou um estudo para comparar a influência de três tipos de cavidades de acesso endodôntico: convencional, conservadora e ultraconservadora. Embora um pequeno número de espécimes tenha sido incluído no presente estudo, os resultados demonstram que uma cavidade de acesso convencional está enfraquecendo a resistência à fratura de dentes com tratamento endodôntico e que cavidades de acesso conservadoras e ultraconservadoras aumentam a resistência à fratura e a força dos dentes tratados.

Rover e Belladonna (2017) realizaram um estudo e os resultados não mostraram benefícios associados as CECs, esse tipo de acesso em molares superiores resultou em menor detecção dos canais radiculares e não aumentou a resistência a fratura.

Silva e colaboradores (2019) avaliaram a influência das cavidades endodônticas ultraconservadoras e não houve verdadeiro benefício associado, elas resultaram em mais acúmulo de detritos dentro dos canais radiculares, dificultou o procedimento de limpeza da câmara pulpar, aumentando o tempo total necessário para a realização do tratamento do canal radicular, e não foi associada a um aumento na resistência à fratura de pré-molares superiores com 2 raízes com preenchimento radicular.

Para Silva e Nery (2019), a confecção de AEMI pode influenciar no sucesso do tratamento endodôntico, pois suas características de preparo influenciam na localização de canais radiculares, principalmente o MV2 em molares superiores. A instrumentação dos canais radiculares pode ser comprometida, principalmente os canais de raízes palatinas em molares superiores e canais de raiz distal de molares inferiores.

Para Augusto et al., (2020) as cavidades de acesso endodôntico ultraconservador não oferecem nenhuma vantagem em comparação com as cavidades de acesso endodôntico tradicionais na capacidade de moldar canais, nem na resistência à fratura de molares inferiores.

Clark e Khademi são considerados os precursores das cavidades de acesso minimamente invasivos em endodontia. Há uma década publicaram o primeiro artigo descrevendo um novo conceito para a realização de cavidades endodônticas. Apesar da grande quantidade de dados científicos publicados sobre, a comparação dos benefícios e danos entre essas cavidades de acesso minimamente invasivos e as cavidades de acesso tradicionais permanece discutível. 


\section{Considerações Finais}

Considerando dados disponíveis até o momento, os acessos endodônticos minimamente invasivos não ofereceram quaisquer vantagens em comparação com cavidades de acesso endodôntico convencional, inclusive faltam evidências que os acessos endodônticos minimamente invasivos promovam maior resistência ao elemento dentário, pois a fratura dentária é multifatorial e depende também de outros fatores. Portanto, uma técnica deve provar seu benefício irrefutável, sobretudo, sua segurança. Além disso, os acessos endodônticos minimamente invasivos podem interferir nos procedimentos subsequentes, como a localização dos canais radiculares, limpeza, instrumentação e obturação.

O acesso contemporâneo é uma alternativa a se lançar mão, pois é conduzido pela anatomia e morfologia do sistema de canais radiculares que objetiva a preservação de estrutura dentária sem desconsiderar bases científicas e biológicas da Endodontia. Dessa forma, destaca-se a importância de mais estudos e pesquisas abordando os acessos contemporâneos, possibilitando ao profissional uma alternativa segura para a realização de um tratamento endodôntico eficaz. Sugerem-se também novas pesquisas, que analisem a resistência dos dentes com acessos endodônticos minimamente invasivos e acessos convencionais e que comparem as diferentes técnicas.

\section{Referências}

Albuquerque, L. A., Archer, C. E., Souza, R. M. S., Travassos, R. M. C., Gomes, S. G. F., \& Santos, R. A. (2012). Prevalência de doenças pulpares e periapicais na Clínica de especialização em Endodontia da FOP/UPE. Revista de cirurgia e traumatologia buco-maxilo-facial, 11(1), 9-12. revistacirurgiabmf.com/2019/03/Artigos/RevistaV19N3.pdf.

Augusto, C. M., Barbosa, A. F. A., Guimarães, C. C., Lima, C. O., Ferreira, C. M., Sassone, L. M., \& Silva, E. J. N. L. (2020). Um estudo de laboratório do impacto de cavidades de acesso ultraconservadoras e raiz mínima canal diminui na capacidade de moldar canais em molares inferiores extraídos e sua fratura resistência. International Endodontic Journal, 53 (11), 1516-1529. 10.1111 / iej.13369

Barbosa, A. F. A., Silva, E. J. N. L., Coelho, B. P., Ferreira, C. M. A., Lima, C. O. \& Sassone, L. M. (2020). A influência do desenho da cavidade de acesso endodôntico na eficácia da instrumentação do canal, redução microbiana, preenchimento do canal radicular e resistência à fratura em molares inferiores. International Endodontic Journal, 53 (12), 1666-1679. https://doi: 10.1111 / iej.13383.

Bóveda, C., \& Kishen, A. (2015). Contracted endodontic cavities: the foundation for less invasive alternatives in the management of apical periodontitis. Endodontic Topics, 33 (1), 169-186. https://onlinelibrary.wiley.com/doi/abs/10.1111/etp.12088.

Bürklein, S. \& Schäfer E. (2015). Minimally invasive endodontics. Quintessence International, $46 \quad$ (2), 119-24. http://www.quintpub.com/userhome/qi/qi_2015_02_s0119.pdf.

Connert, T., Zehnder, M. S., Amato, M., Weiger, R., Kuhl, S., \& Krastl, G. (2017). Microguided Endodontics: a method to achieve minimallyinvasive access cavity preparation and root canal location in mandibular incisors using a novel computer-guided technique. Switzerland. International Endodontic Journal, 51 (2), 247-55. https://doi.org/10.1111/iej.12809.

Carvalho, N. K., Monteiro, A. G. V., Santo, L. F. E. \& Porto, A. R. (2020). Acesso minimamente invasivo: revisão de literatura. Ciência atual, 15 (1), $74-81$. http://www.cnad.edu.br/revistacienciaatual/index.php/cafsj/article/view/401/pdf .

Gluskin, A. H., Peters, C. I. \& Peters, O. A. (2014). Minimally invasive endodontics: challenging prevailing paradigms. British Dental Journal, 216 (6), $347-$ 353. http://www.cnad.edu.br/revista-ciencia-atual/index.php/cafsj/article/view/401/pdf.

Lima, C. O., Barbosa, A. F. A., Ferreira, C. M., Augusto, C. M., Sassone, L. M., Lopes, R. T., Fidel, S. R. \& Silva, E. J. N. L. (2020). O impacto das estratégias de preparação do canal radicular minimamente invasivas na capacidade de moldar os canais radiculares dos molares inferiores. International Endodontic Journal, 53 (12), 1680-1688. https://onlinelibrary.wiley.com/doi/abs/10.1111/iej.13384 .

Marinescu, A. G., Cîrligeriu, L. E., Pușcu, S. A. B., Horhat, R. M., Sgîia, S. T., Stoia, D. I., \& Nica, L. M. (2020). Avaliação da resistência de fratura dos dentes com diferentes projetos de cavidades de acesso endodôntico. Jornal Romeno de Reabilitação Oral, 12 (2), 76-84. https://www.rjor.ro/wpcontent/uploads/2020/06/FRACTURE-STRENGTH-EVALUATION-OF-TEETH-WITH-DIFFERENT-DESIGNS-OF-ENDODONTIC-ACCESS-

CAVITIES.pdf .

Mukherjee, P., Patel, A., Chandak, M., \& Kashikar, R. (2017). Minimally Invasive Endodontics a Promising Future Concept: A ReviewArticle. International Journal of Scientific Study, 5 (1), 245-251. https://www.ijss-sn.com/uploads/2/0/1/5/20153321/ijss_apr_ra02_-_2017.pdf .

Plotino, G., Grande, N. M., Isufi, A., Ioppolo, P., Pedulla, E., Bedini, R., Gambarini, G., \& Testarelli, L. (2017). Resistência à fratura de dentes tratados endodonticamente com diferentes designs de cavidade de acesso. Journal of Endodontics, 43 (6), 995-1000.http://dx.doi.org/10.1016/j.joen.2017.01.022.

Rover, G., Belladonna, F. G., Bortoluzzi, E. A., De-Deus, G., Silva, E. J. N. L., \& Teixeira, C. S. (2017). Influência do projeto de cavidade de acesso na detecção do canal radicular, eficácia da instrumentação e resistência à fratura avaliada em molares maxilares. Journal of Endodontics, 43 (10), $1657-1662$. https://www.academia.edu/34000424/Influence_of_Access_Cavity_Design_on_Root_Canal_Detection_Instrumentation_Efficacy_and_Fracture_Resistance_ Assessed_in_Maxillary_Molars?auto=citations\&amp;from=cover_page . 
Research, Society and Development, v. 10, n. 15, e28101522407, 2021

(CC BY 4.0) | ISSN 2525-3409 | DOI: http://dx.doi.org/10.33448/rsd-v10i15.22407

Rover, G., Lima, C. O., Belladonna, F. G., Garcia, L. F. R., Bertoluzzi, E. A., Silva, E. J. N. L., \& amp; Teixeira C. S. (2020). Influence of minimallyinvasive endodontic access cavities on root canal shaping and filling ability, pulp chamber cleaning and fracture resistance of extractedhuman mandibular incisors. International Endodontic Journal, 53 (11), 1530-9. https://doi.org/10.1111/iej.13378 .

Silva, A. A., Belladonna, F. G., Rover, G., Lopes, R. T., Moreira, E. J. L., De-Deus, G., \& Silva E. J. N. L. (2020). O acesso ultraconservador afeta a eficácia do tratamento do canal radicular e a resistência à fratura de pré-molares superiores com duas raízes?. International Endodontic Journal, 53 (2), 265-275. https://onlinelibrary.wiley.com/doi/abs/10.1111/iej.13219.

Silva, E. J. N. L., Barbosa, A. F. A., Attademo, R. S., Lima, C. O., Decurcio, D. A., \& Pereira, L. A. P. (2021). Acessos endodônticos: o que todo endodontista deve saber. Dental Press Endodontic, 11 (1), 16-28. https://doi.org/10.14436/2358-2545.11.1.016-028.oar.

Silva, E. J. N. L., Pinto, K. P., Ajuz, N. C., \& Sassone, L. M. (2021). Dez anos de cavidades de acesso minimamente invasivas em Endodontia:uma análise bibliométrica dos 25 estudos mais citados. Restor Dent Endod, 46 (3). https://doi.org/10.5395/rde.2021.46.e42 .

Silva E. J. N. L., Versiani M. A., Souza E. M. \& De-Deus G. (2021). Cavidades de acesso minimamente invasivas: o tamanho realmente importa?. International Endodontic Journal, 54 (2), 153-155. https://www.researchgate.net/publication/348551634 .

Silva, P. Á. C., \& Silva, I. S. N. (2019). Acesso endodôntico minimamente invasivo: revisão de literatura. Salusvita, 38 (1), 195-212. https://secure.unisagrado.edu.br/static/biblioteca/salusvita/salusvita_v38_n1_2019/salusvita_v38_n1_2019_art_13.pdf .

Vasconcellos, R. C. C., Barbosa, V. F., Inojosa, I. F. A. J., Machado, J. L., Santos, R. A., Menezes, R. F. \& Soares, N. M. L. T. (2012). Influence of coronal opening in the location of root canals in mandibular incisors. Dental Press Endod, 2 (3), 74-9. https://www.dentalpresspub.com/en/endo/v02n3/74.pdf .

Tzimpoulas, N. E., Alisafis, M. G., Tzanetakis, G. N. \& Kontakiotis, E. G. (2012). A prospective study of the incidence of extraction andretention of endodontically treated teeth with uncertain prognosis after endodontic referral. Journal of Endodontics, 38 (10), 1326- 9.https://38 (10): 1326-9. 10.1016. 